\title{
Continuously tunable photonic radio frequency and microwave orthogonally polarized single sideband generator using Kerr micro-combs
}

David Moss ( $\sim$ dmoss@swin.edu.au )

Swinburne University of Technology https://orcid.org/0000-0001-5195-1744

\section{Research Article}

Keywords: Micro-ring resonator, radio frequency, single sideband modulation

Posted Date: November 4th, 2021

DOI: https://doi.org/10.21203/rs.3.rs-1048646/v1

License: (c) (i) This work is licensed under a Creative Commons Attribution 4.0 International License.

Read Full License 


\title{
Continuously tunable photonic radio frequency and microwave orthogonally polarized single sideband generator using Kerr micro-combs
}

\author{
David J. Moss \\ Optical Science Centre, Swinburne University of Technology, Hawthorn, VIC 3122, Australia
}

E-mail: dmoss@swin.edu.au

\begin{abstract}
We demonstrate a continuously RF tunable orthogonally polarized optical single sideband (OP-OSSB) generator based on dual cascaded micro-ring resonators. By splitting the input double sideband signal into an orthogonally polarized carrier and lower sideband via TE- and TM-polarized MRRs, an OP-OSSB signal is generated. A large tuning range of the optical carrier to sideband ratio of up to $57.3 \mathrm{~dB}$ is achieved by adjusting the polarization angle of the input light. The operation RF frequency of the OP-OSSB generator can be continuously tuned with a $21.4 \mathrm{GHz}$ range via independent thermal control of the two MRRs. Our device represents a competitive approach towards OP-OSSB generation with wideband tunable RF operation, and is promising for photonic RF signal transmission and processing in radar and communication systems.
\end{abstract}

Keywords: Micro-ring resonator, radio frequency, single sideband modulation

\section{Introduction}

Microwave photonics has attracted great interest in a wide range of applications in radar and communications systems [1-4] due to its numerous intrinsic advantages such as broad RF operation bandwidth, low loss, and strong immunity to electromagnetic interference [5-8]. As one of the key technologies of microwave photonic systems, modulation formats have a significant impact on the overall system performance [9-11]. Offering advantages such as overcoming dispersion-induced distortion, enhanced spectral efficiency, and the ability to separately manipulate the optical carrier and sidebands via polarization-sensitive optical components, orthogonally polarized optical single sideband (OP-OSSB) modulation has been widely exploited in applications ranging from antenna beamforming to microwave photonic signal processing [12-16].

Many approaches have been demonstrated to realize OPOSSB generation, including using specially designed modulators such as acoustic optical modulators [17], Sagnacloop-based modulators [18], dual-polarization quadrature phase shift keying modulators [19], and polarization modulators [20]. In other approaches, differential group delay elements were used to cross-polarize the optical carrier and the sideband by exploiting birefringence $[15,16]$. In addition, fiber-based stimulated Brillouin scattering has been used to control the polarization of optical signals to achieve OP-OSSB generation $[21,22]$. However, the above approaches face limitations of one form or another. On the one hand, RF couplers can introduce an electrical bandwidth bottleneck for the entire system, while fiber-based methods face limitations 


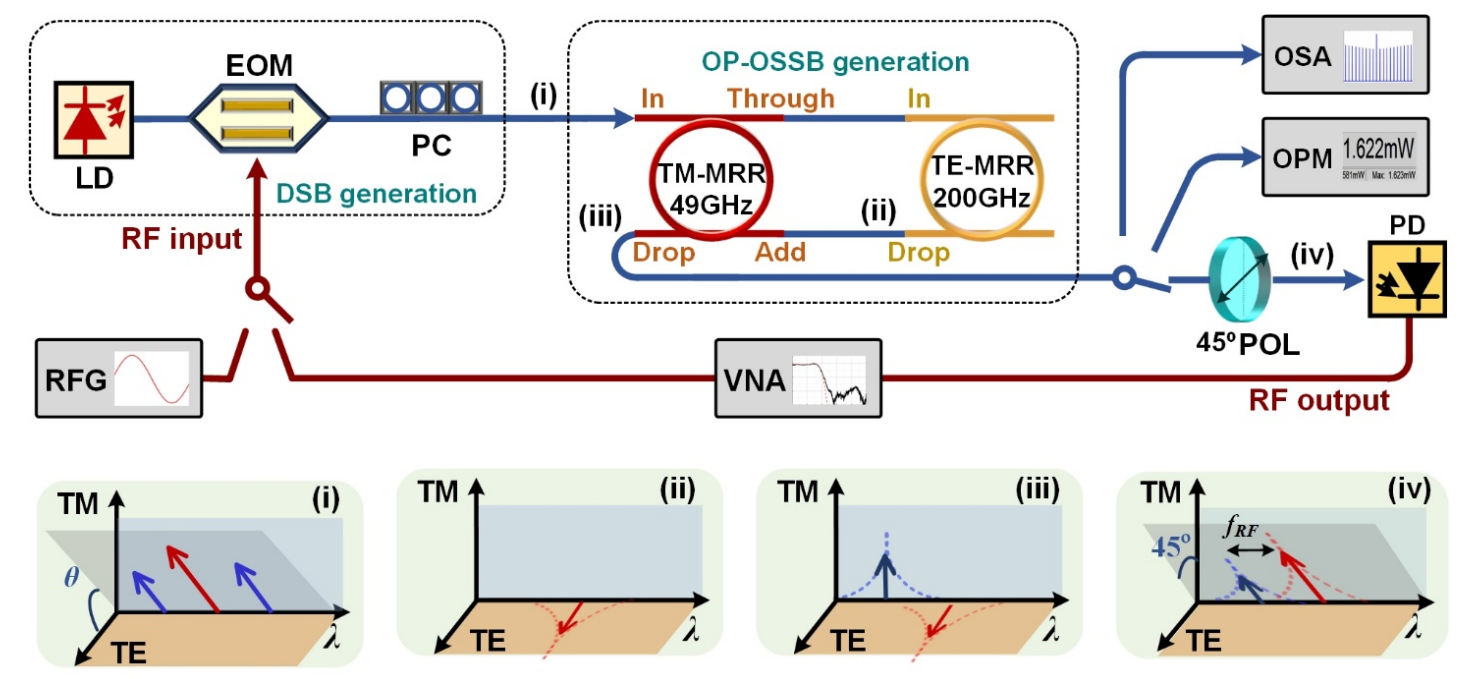

Fig. 1. Schematic of the proposed orthogonally polarized optical single sideband (OP-OSSB) generator. LD: laser diode. EOM: electro-optical modulator. PC: polarization controller. DSB: double sideband. OSA: optical spectrum analyzer. OPM: optical power meter. $45^{\circ}$ POL: optical polarizer with the polarization direction having an angle of $45^{\circ}$ to the TM axis. PD: photodetector. VNA: vector network analyzer. RFG: RF generator.

in terms of footprint and stability, important for moving the technology beyond the laboratory.

Integrated microwave photonics is a competitive solution to address these challenges [23], offering a reduced footprint and higher reliability [24]. Recently, we reported an OPOSSB generator that operated at fixed RF frequencies based on a micro-ring resonator (MRR) that supported both TE- and TM-polarizations [25]. Here, we report a continuously tunable (in RF frequency) OP-OSSB generator based on dual cascaded integrated MRRs. The operation RF frequency of the OPOSSB generator, determined by the spectral interval between the TE and TM resonances, can be dynamically tuned via separate thermo-optical control of the two MRRs, resulting in operation over a wide RF tuning range. Moreover, by controlling the polarization angle of the input light, a large dynamic tuning range in the optical carrier-to-sideband ratio (OCSR) of up to $57.3 \mathrm{~dB}$ is demonstrated.

\section{Operation principle}

Figure 1 shows a schematic of the wideband tunable OPOSSB generator. Continuous-wave light from a tunable laser source is intensity-modulated by an RF signal to generate a double sideband signal with a polarization angle $\theta$ to the TEaxis (Fig. 1(i)), and then fed into the two cascaded MRRs that support TE- and TM-polarization modes. When the wavelength of the optical carrier and the input RF frequency match with the orthogonally polarized resonances of the two MRRs, the optical carrier and one sideband of the double sideband signal can be dropped (Fig. 1(ii)). After that, the dropped optical carrier and sideband are combined together by connecting the drop-port of the second $(200 \mathrm{GHz}$, TE polarization) MRR to the add-port of the first (49GHz, TM) MRR (Fig. 1(iii)), thus achieving OP-OSSB modulation.

To analyze the polarization states of our device, we use the Jones matrix formalism. While other methods exist, such as the Poincaré sphere and Stokes parameters [26], we use the Jones matrix for simplicity since the polarization eigenmodes of the MRRs serve as a natural basis. The transmission of the dual MRRs can be written as

$$
R=\left[\begin{array}{ll}
D_{T E} & 0 \\
0 & D_{T M}
\end{array}\right]
$$

where $D_{\mathrm{TE}}$ and $D_{\mathrm{TM}}$ are the drop-port transfer functions of the $49 \mathrm{GHz}$ (TE) MRR and $200 \mathrm{GHz}$ (TM) MRR given by

$$
\begin{aligned}
D_{T E} & =\frac{-k_{T E}{ }^{2} \sqrt{a_{T E}} e^{i \phi_{T E} / 2}}{1-t_{T E}{ }^{2} a_{T E} e^{i \phi_{T E}}} \\
D_{T M} & =\frac{-k_{T M}{ }^{2} \sqrt{a_{T M}} e^{i \phi_{T M} / 2}}{1-t_{T M}{ }^{2} a_{T M} e^{i \phi_{T M}}}
\end{aligned}
$$

where $t_{T E}, t_{T M}, k_{T E}$ and $k_{T M}$ are the field transmission and crosscoupling coefficients between the bus waveguide and the ring ( $t^{2}+k^{2}=1$ for lossless coupling), $a_{T E}$ and $a_{T M}$ represent the round-trip transmission factors, $\phi_{\mathrm{TE}}=2 \pi L_{\mathrm{TE}} \times n_{\mathrm{eff}} \mathrm{TE} / \lambda$ and $\phi_{\mathrm{TM}}=2 \pi L_{\mathrm{TM}} \times n_{\text {eff_TM }} / \lambda$ are the single-pass phase shifts of the TE-MRR and TM-MRR, respectively, with $L_{T E}$ and $L_{T M}$ denoting the round-trip length, $n_{\text {eff_TE }}$ and $n_{\text {eff_TM }}$ denoting the effective indices, and $\lambda$ denoting the wavelength. 


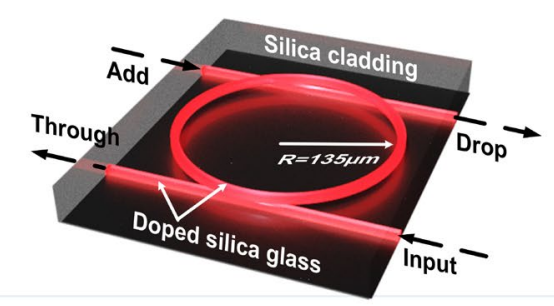

(a)

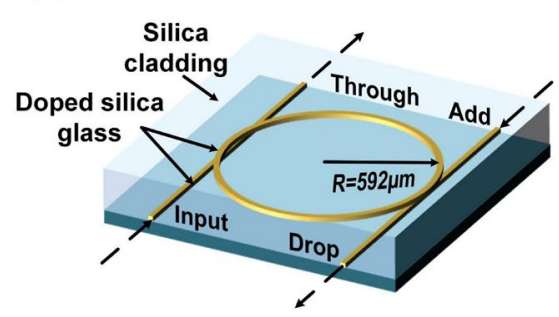

(d)
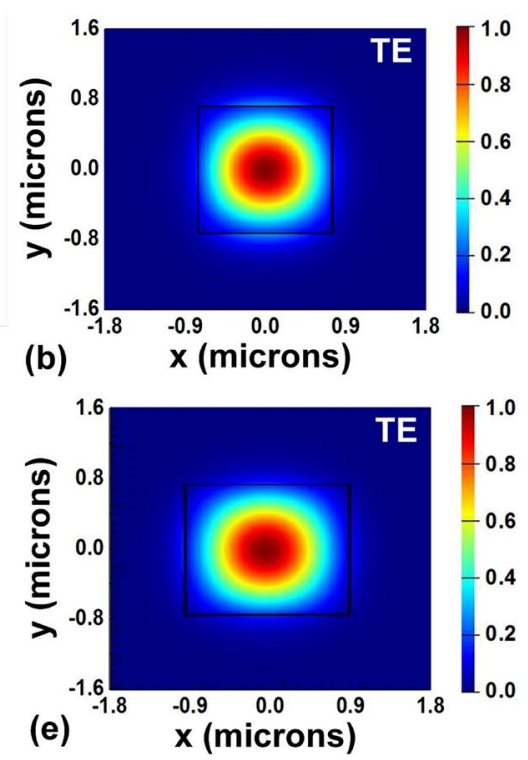

(c)
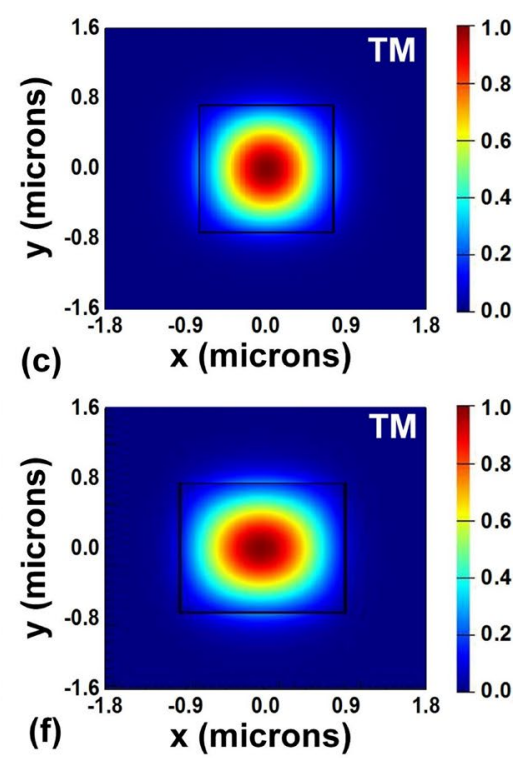

Fig. 2. (a) Schematic illustration of the 200GHz FSR MRR, (b) TE and (c) TM mode profiles of the 200GHz MRR. (d) Schematic illustration of the 49GHz FSR MRR, (e) TE and (f) TM mode profiles of the 49GHz FSR MRR.

For a general optical field input $E_{0}\left[\begin{array}{c}\cos \theta \\ \sin \theta\end{array}\right]$, the output field of the dual MRRs is

$$
E_{\text {out }}=R E_{0}\left[\begin{array}{c}
\cos \theta \\
\sin \theta
\end{array}\right]=E_{0}\left[\begin{array}{l}
D_{T E} \cdot \cos \theta \\
D_{T M} \cdot \sin \theta
\end{array}\right]
$$

As reflected by the above equation, the optical power of the spectral components dropped by the $49 \mathrm{GHz}$ (TE) MRR and $200 \mathrm{GHz}$ (TM) MRR are proportional to $\cos ^{2} \theta$ and $\sin ^{2} \theta$, respectively. Thus, the OCSR (with the $49 \mathrm{GHz}$ MRR for the carrier and the $200 \mathrm{GHz}$ MRR for the sideband) is given by

$$
\operatorname{OCSR}(\theta) \propto \cot ^{2} \theta
$$

which can be continuously tuned by adjusting $\theta$. Since $\cot ^{2} \theta$ can infinitely approach 1 or 0 as $\theta$ approaches 0 or $\pi / 2$, a large

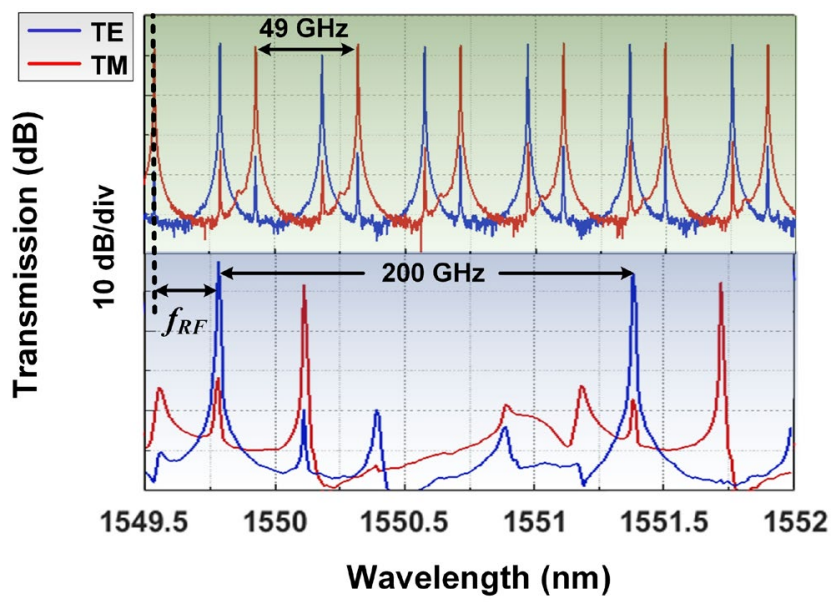

Fig. 3. Measured transmission spectra of the (49GHz FSR MRR) and $200 \mathrm{GHz}$ FSR MRR. tuning range of the OCSR can be produced. Moreover, the generated OP-OSSB signal can be converted back into the RF domain by passing it through an optical polarizer (Fig. 1(iv)). The operation RF frequency of the proposed OP-OSSB generator is determined by the spectral interval between adjacent resonances of the $49 \mathrm{GHz}$ and $200 \mathrm{GHz}$ MRR. Thus, with separate thermal controls of the MRRs, tunable OPOSSB generation over a wide RF tuning range can be realized.

\section{Experimental results}

We fabricated $49 \mathrm{GHz}$ FSR and $200 \mathrm{GHz}$ FSR integrated MRRs that each support both TE- and TM- polarization modes, utilizing the TM-polarized resonances of the first MRR (49GHz), and the TE-polarized resonances of the second $(200 \mathrm{GHz})$ MRR. Figures 2(a, d) show a schematic of the two MRRs used in our experiment, which were both fabricated on a high-index doped silica glass platform using CMOS compatible fabrication processes [27-29]. First, highindex ( $\mathrm{n}=\sim 1.70$ at $1550 \mathrm{~nm}$ ) doped silica glass films were deposited using standard plasma enhanced chemical vapour deposition, then patterned using deep UV photolithographically and etched via reactive ion etching to form waveguides with exceptionally low surface roughness [3032]. Finally, silica glass ( $\mathrm{n}=\sim 1.44$ at $1550 \mathrm{~nm}$ ) was deposited as an upper cladding. The waveguides were designed to feature a nearly symmetric cross-section $(1.5 \mu \mathrm{m} \times 2 \mu \mathrm{m}$ for the $49 \mathrm{GHz}$ ring resonator [33] and $1.45 \mu \mathrm{m} \times 1.5 \mu \mathrm{m}$ for the $200 \mathrm{GHz}$ ring resonator [29]), enabling both MRRs to support both TE and TM modes. The calculated TE and TM mode 

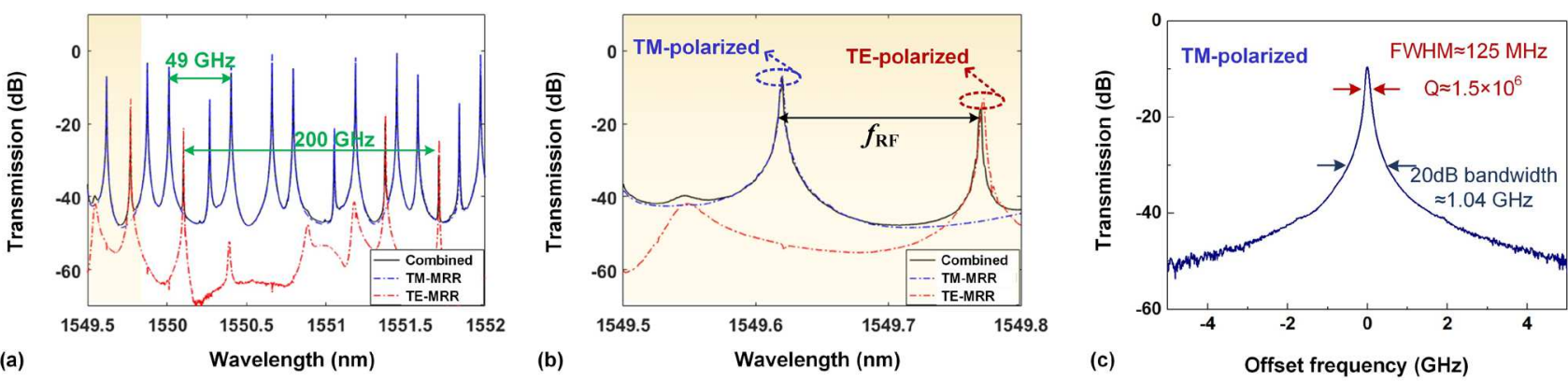

Fig. 4. Measured (a) transmission spectra of the $49 \mathrm{GHz}$ (TM) MRR, 200GHz FSR (TE) MRR, and the combined OP-OSSB generator. (b) Zoom-in spectra of (a) with one TE polarized resonance and one TM polarized resonance. (c) Transmission spectra around one TM-polarized resonance of the 49GHz FSR MRR.

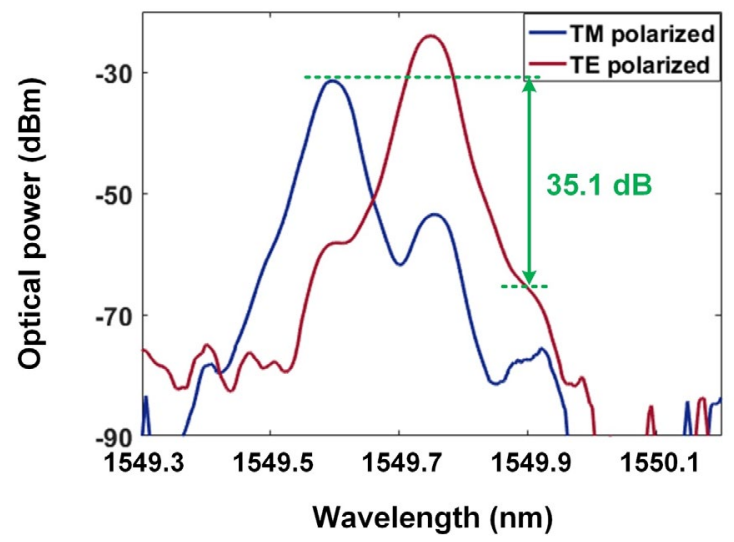

Fig. 5. Optical spectra of the generated orthogonally polarized OSSB signal.

profiles of the MRRs are shown in Figs. 2(b-c, e-f). The difference between the effective indices of the TE and TM modes (49GHz MRR: 1.627 for the TE mode and 1.624 for the TM mode, 200GHz: 1.643 for the TE mode and 1.642 for the TM mode) resulted in slightly different free spectral ranges (FSRs) for the TE and TM resonances and a wide spectral interval between them in the optical communications band (TE/TM separations of $\sim 16.8 \mathrm{GHz}$ and $\sim 41.2 \mathrm{GHz}$ for the $49 \mathrm{GHz}$ and $200 \mathrm{GHz}$ MRRs, respectively). The radius of the $49 \mathrm{GHz}$ MRR was $\sim 592 \mu \mathrm{m}$, corresponding to an FSR of $\sim 0.4$ $\mathrm{nm}[34,35]$ with a $\mathrm{Q}$ factor of $1.5 \times 10^{6}$, while the $200 \mathrm{GHz}$ MRR had a radius of $\sim 135 \mu \mathrm{m}$, corresponding to an FSR of $\sim 1.6 \mathrm{~nm}$ [36-39] and a Q factor of $1.2 \times 10^{6}$, which reduced leakage of the undesired sideband from the $200 \mathrm{GHz}$ MRR's unused resonances. The through-port insertion loss was $\sim 1.5$ $\mathrm{dB}$ after being packaged with fiber pigtails via butt coupling and employing on-chip mode converters. The devices have been shown to exhibit negligible nonlinear loss (two photon absorption) up to $25 \mathrm{GW} / \mathrm{cm} 2$ [40]. The measured transmission spectra of the two MRRs are shown in Fig. 3, in which the FSRs and operation RF frequency are clearly reflected.

The two MRRs were then connected via polarization maintaining fiber pigtails with the 49GHz MRR's throughport being connected to the $200 \mathrm{GHz}$ MRR's input. Both of the MRRs' drop-ports were then combined by connecting the drop-port of the $200 \mathrm{GHz}$ (TE) MRR to the add-port of the $49 \mathrm{GHz}$ (TM) MRR. Figure 4 shows the measured transmission spectra of the dual MRRs. As reflected by the dual resonances, both of the MRRs supported dual polarization modes. The first (49 GHz FSR) and second MRR (200GHz FSR) served as TM- and TE-MRRs for the OPOSSB generation, respectively. The operation RF frequency was determined by the spectral interval between adjacent orthogonally polarized resonances (Fig. 4(b)). The $49 \mathrm{GHz}$ MRR featured a high $\mathrm{Q}$ factor, corresponding to a $20 \mathrm{~dB}$ -
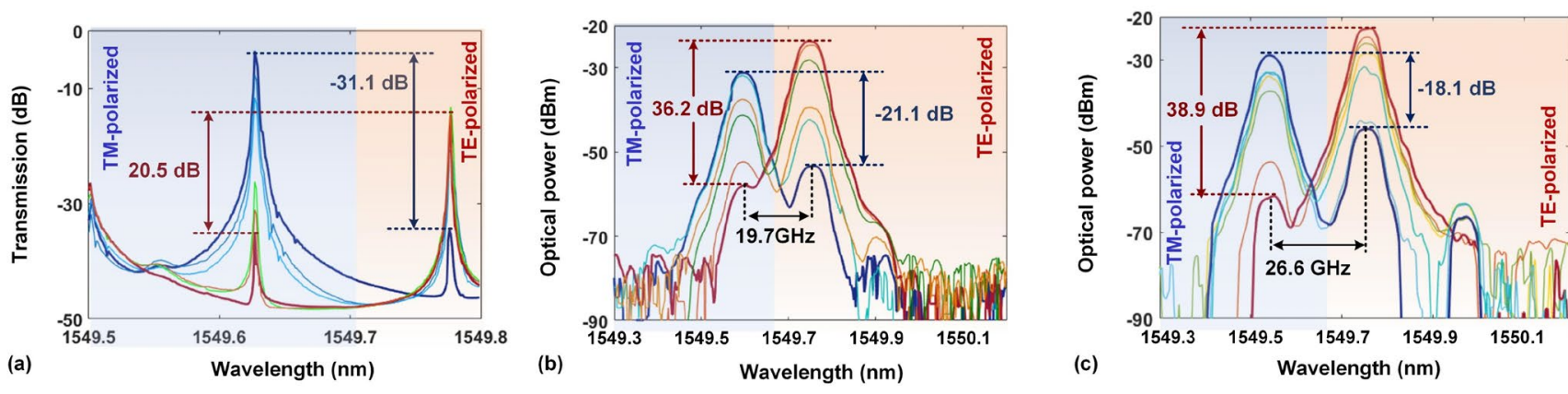

Fig. 6. Measured (a) transmission spectra of the dual MRRs and (b-c) optical spectra of the generated orthogonally polarized OSSB signal with continuously tunable OCSR. 

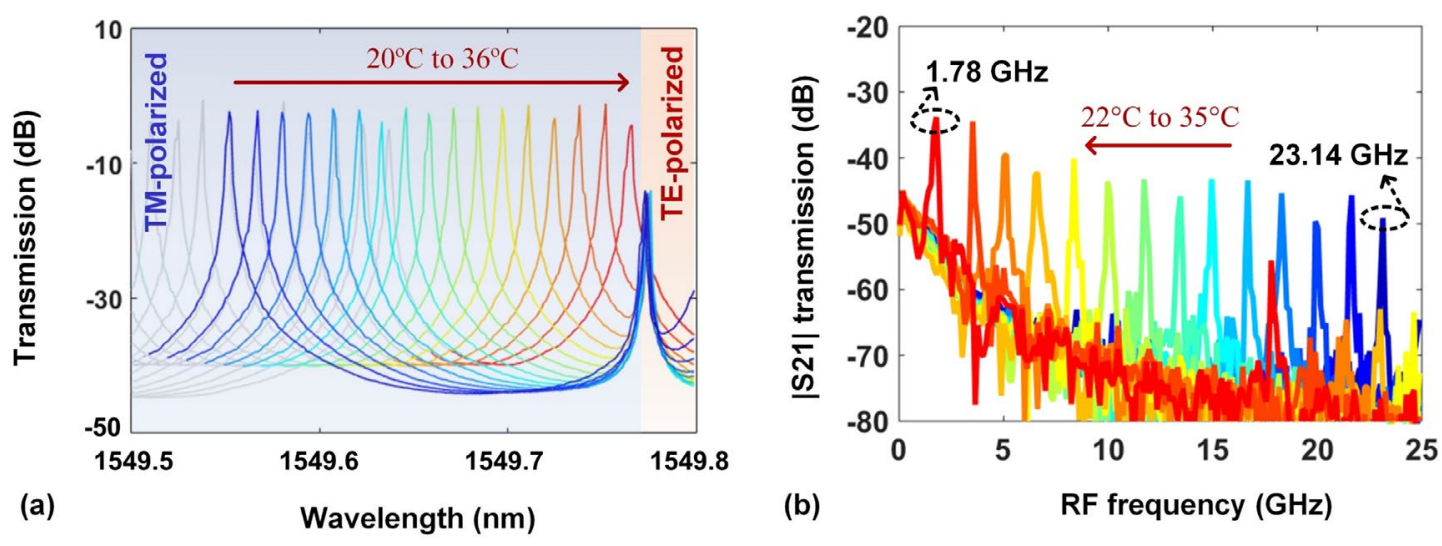

Fig. 7. Measured (a) optical transmission spectra, and (b) RF transmission response of the OP-OSSB generator with thermo-optical control.

bandwidth of $\sim 1.04 \mathrm{GHz}$ (Fig. 4(b)), indicating a high rejection ratio of the optical carrier and lower accessible RF frequency down to the sub-GHz level for the OP-OSSB generator.

During the experiment, we tuned the carrier wavelength to one of the TE resonances of the $200 \mathrm{GHz}$ MRR centered at $\sim 1549.78 \mathrm{~nm}$, and drove an intensity modulator (ixBlue) with an RF signal such that the lower sideband could be dropped by the adjacent TM resonance of the $49 \mathrm{GHz}$ MRR. The orthogonally polarized carrier and lower sideband were obtained at the output of the dual MRRs, where the optical power of the upper sideband was suppressed by over $35 \mathrm{~dB}$ as compared with that of the lower sideband, as shown in Fig. 5.

As mentioned, the ratio between the orthogonally polarized optical carrier and lower sideband could be tuned via the input polarization angle ( $\theta$ in Fig. 1). We measured the corresponding transmission of the dual MRRs (Fig. 6(a)) as $\theta$ was varied. The extinction ratio between $\mathrm{TE}$ and $\mathrm{TM}$ resonances varied from $20.5 \mathrm{~dB}$ to $-31.1 \mathrm{~dB}$, corresponding to a dynamic tuning range of up to $51.1 \mathrm{~dB}$ for the OSCR. Figures 6(b)-(c) show the optical spectra of the generated OPOSSB signal with input RF frequencies at $19.7 \mathrm{GHz}$ and 26.6 $\mathrm{GHz}$. Continuously variable OCSRs ranging from -21.1 to $36.2 \mathrm{~dB}$ and from -18.1 to $38.9 \mathrm{~dB}$ were achieved for the 19.7
GHz- and 26.6 GHz- RF input, respectively, yielding a large OCSR tuning range of up to $57.3 \mathrm{~dB}$ for our OP-OSSB generator. We note that the orthogonal polarization modes of the cascaded MRRs could also potentially provide an additional control dimension for optical logic gates, thus serving as a promising candidate for optical computing functions $[41,42]$.

To achieve wide RF tunability, we varied the spectral interval between the TE resonances of the $200 \mathrm{GHz}$ MRR and the TM resonances of the $49 \mathrm{GHz}$ MRR by applying separate thermal controls to two devices [43]. The temperature of the $49 \mathrm{GHz}$ (TM) MRR was varied from $20^{\circ} \mathrm{C}$ to $36^{\circ} \mathrm{C}$ while the temperature of the $200 \mathrm{GHz}$ (TE) MRR was maintained at 25 ${ }^{\circ} \mathrm{C}$. Figure 7(a) shows the measured transmission spectra of the dual MRRs as a function of temperature, where the $49 \mathrm{GHz}$ MRR TM polarized resonance was thermally tuned over a range of $0.2 \mathrm{~nm}$ while the $200 \mathrm{GHz}$ MRR TE-polarized resonance was fixed, thus leading to over $20 \mathrm{GHz} \mathrm{RF}$ tuning range for our OP-OSSB generator. To reflect the wide RF operation of our approach, the OP-OSSB signal was converted into single polarization via a polarizer and detected by a photodetector. The RF transmission response of the system was measured by a vector network analyzer. As shown in Fig. 7(b), wideband RF operation up to $23.14 \mathrm{GHz}$ was
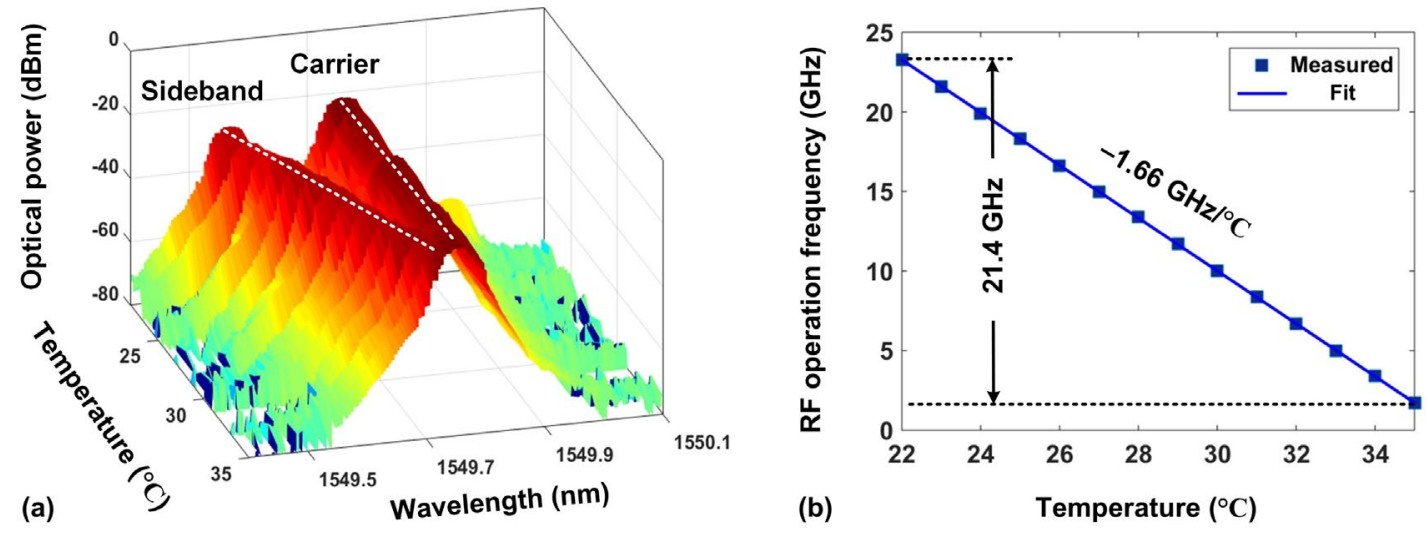

Fig. 8. (a) Optical spectra and (b) extracted operation RF frequency of the generated OP-OSSB signals with thermal tuning. 
demonstrated. The optical spectra of the OP-OSSB signals with tunable RF operation were measured and shown in Fig. 8 (a). As the chip temperature of the $49 \mathrm{GHz}$ (TM) MRR was varied from $22{ }^{\circ} \mathrm{C}$ to $35^{\circ} \mathrm{C}$, the RF operation frequency of the OP-OSSB generator changed from $1.81 \mathrm{GHz}$ to $23.27 \mathrm{GHz}$ with a fit slope of $-1.66 \mathrm{GHz} /{ }^{\circ} \mathrm{C}$ (Fig. 8(b)), thus confirming the wide tuning range of our approach. The RF operational bandwidth of our OP-OSSB generator was limited by the thermal controller $\left(\sim 15{ }^{\circ} \mathrm{C}\right.$ tuning range). Covering the entire FSR of the TM-MRR (49 GHz) would only require a thermal tuning range of $29.5^{\circ} \mathrm{C}$ which is readily achievable [43]. We note that the small change in FSR with temperature can easily be compensated for by the calibration of the device. Furthermore, by using multiply FSR spaced TM resonances, the RF tuning range can be increased arbitrarily, reaching even the $\mathrm{THz}$ region — well beyond that of electrical approaches [44].

Since the cascaded micro-ring resonators are passive filtering devices, they did not contribute to the performance of the generated orthogonally polarized optical single sideband signals in terms of coherence/dephasing time. The dephasing time of the generated signals in our case was mainly determined by the coherence length $L_{\mathrm{coh}}$ of our laser, given by [45]

$$
L_{c o h}=\sqrt{\frac{2 \ln 2}{\pi n}} \frac{\lambda^{2}}{\Delta \lambda}
$$

where $\lambda$ is the central wavelength of the source $(\sim 1550 \mathrm{~nm})$, $n$ is the refractive index of fiber $(\sim 1.45)$, and $\Delta \lambda$ is the full width at half maximum (FWHM) spectral width of the source. Our laser (TUNICS T100S-HP) had a $400 \mathrm{kHz}$ FWHM spectral width, yielding a coherence length of $\sim 414 \mathrm{~m}$.

Here, we employed high-Q MRRs for the OP-OSSB generation, which support a narrow instantaneous RF bandwidth. For RF applications requiring a broad instantaneous bandwidth, either a lower Q MRR [46] or a higher order filter [47-52] can be employed to replace the high-Q TM-MRR in our experiment. The former can yield a $3 \mathrm{~dB}$ bandwidth from 2 to $12 \mathrm{GHz}$ corresponding to a $\mathrm{Q}$ factor ranging from 60,000 to 10,000 , while the latter can achieve a $3 \mathrm{~dB}$ bandwidth of $100 \mathrm{GHz}$ or even higher. It should also be noted that the two MRRs can be further integrated on the same chip, with the spectral interval tuned by employing separate thermo-optical micro-heaters [53]. The success of this CMOS compatible platform for nonlinear optics arises from its negligible two photon absorption that, whilst it has been shown to be beneficial in some cases [54, 55], poses a barrier to generating optical Kerr micro-combs in integrated microresonators[28]. The Hydex platform has been extremely successful at microcomb generation for applications, such as here, to microwave and radio frequency photonics [55-113] and even quantum optical applications [114-122].

\section{Conclusion}

We propose and experimentally demonstrate an orthogonally polarized optical single sideband (OP-OSSB) generator based on dual integrated MRRs. By splitting the input double sideband signal into an orthogonally polarized carrier and lower sideband via TE- and TM-polarized MRRs, an OP-OSSB signal can be generated. A large tuning range for the optical carrier to sideband ratio of up to $57.3 \mathrm{~dB}$ was achieved by adjusting the polarization angle of the input light. The operational radio frequency of the OP-OSSB generator could be widely varied via separate thermo-optical control of the two MRRs, resulting in a broad RF tuning range of over $21.4 \mathrm{GHz}$. This approach provides a new way to realize OPOSSB generation with wideband tunable RF operation, which is promising for RF photonic signal processing in radar and communication systems.

Competing interests: The authors declare no competing interests.

\section{References}

[1] J. Capmany, and D. Novak, "Microwave photonics combines two worlds," Nat. Photonics 1, 319-330 (2007).

[2] J. P. Yao, "Microwave Photonics," IEEE J. Lightwave Technol. 27, 314-335 (2009).

[3] K. Xu, R. X. Wang, Y. T. Dai, F. F. Yin, J. Q. Li, Y. F. Ji, and J. T. Lin, "Microwave photonics: radio-over-fiber links, systems, and applications [Invited]," Photonics Res. 2, 54-63 (2014).

[4] J. Y. Wu, X. Y. Xu, T. G. Nguyen, T. Moein, S. T. Chu, B. E. Little, R. Morandotti, A. Mitchell, and D. J. Moss, "RF Photonics: An Optical Microcombs' Perspective," IEEE J. Sel. Top. Quant. 24, 1-20 (2018).

[5] V. R. Supradeepa, C. M. Long, R. Wu, F. Ferdous, E. Hamidi, D. E. Leaird, and A. M. Weiner, "Comb-based radiofrequency photonic filters with rapid tunability and high selectivity," Nat. Photonics 6, 186-194 (2012).

[6] R. A. Minasian, "Ultra-Wideband and Adaptive Photonic Signal Processing of Microwave Signals," IEEE J. Quantum Elect. 52 (2016).

[7] X. X. Xue, Y. Xuan, H. J. Kim, J. Wang, D. E. Leaird, M. H. Qi, and A. M. Weiner, "Programmable Single-Bandpass Photonic RF Filter Based on Kerr Comb from a Micro-ring," J. Lightwave Technol. 32, 3557-3565 (2014).

[8] B. Corcoran, T. D. Vo, M. D. Pelusi, C. Monat, D. X. Xu, A. Densmore, R. B. Ma, S. Janz, D. J. Moss, and B. J. Eggleton, "Silicon nanowire based radio-frequency spectrum analyzer," Opt. Express 18, 20190-20200 (2010).

[9] Y. M. Zhang, F. Z. Zhang, and S. L. Pan, "Optical single sideband polarization modulation for radio-over-fiber system and microwave photonic signal processing," Photonics Res. 2, B80B85 (2014).

[10] U. Gliese, S. Norskov, and T. N. Nielsen, "Chromatic dispersion in fiber-optic microwave and millimeter-wave links," IEEE T. Microw. Theory 44, 1716-1724 (1996). 
[11] S. R. Blais, and J. P. Yao, "Optical single sideband modulation using an ultranarrow dual-transmission-band fiber Bragg grating," IEEE Photonic Tech. L. 18, 2230-2232 (2006).

[12] S. L. Pan, and Y. M. Zhang, "Tunable and wideband microwave photonic phase shifter based on a single-sideband polarization modulator and a polarizer," Opt. Lett. 37, 4483-4485 (2012).

[13] L. X. Wang, W. Li, H. Wang, J. Y. Zheng, J. G. Liu, and N. H. Zhu, "Photonic Generation of Phase Coded Microwave Pulses Using Cascaded Polarization Modulators," IEEE Photonic Tech. L. 25, 678-681 (2013).

[14] L. X. Wang, W. Li, J. Y. Zheng, H. Wang, J. G. Liu, and N. H. Zhu, "High-speed microwave photonic switch for millimeterwave ultra-wideband signal generation," Opt. Lett. 38, 579-581 (2013).

[15] Z. H. Li, C. Y. Yu, Y. Dong, L. H. Cheng, L. F. K. Lui, C. Lu, A. P. T. Lau, H. Y. Tam, and P. K. A. Wai, "Linear photonic radio frequency phase shifter using a differential-group-delay element and an optical phase modulator," Opt. Lett. 35, 18811883 (2010).

[16] B. Vidal, T. Mengual, C. Ibanez-Lopez, and J. Marti, “Optical beamforming network based on fiber-optical delay lines and spatial light modulators for large antenna arrays," IEEE Photonic Tech. L. 18, 2590-2592 (2006).

[17] D. Dolfi, P. Joffre, J. Antoine, J. P. Huignard, D. Philippet, and P. Granger, "Experimental demonstration of a phased-array antenna optically controlled with phase and time delays," Appl. Optics 35, 5293-5300 (1996).

[18] J. Y. Zheng, L. X. Wang, Z. Dong, M. Xu, X. Wang, J. G. Liu, N. H. Zhu, S. LaRochelle, and G. K. Chang, "Orthogonal SingleSideband Signal Generation Using Improved Sagnac-LoopBased Modulator," IEEE Photonic Tech. L. 26, 2229-2231 (2014).

[19] W. T. Wang, J. G. Liu, H. K. Mei, and N. H. Zhu, "Phasecoherent orthogonally polarized optical single sideband modulation with arbitrarily tunable optical carrier-to-sideband ratio," Opt. Express 24, 388-399 (2016).

[20] L. Campillo, "Orthogonally polarized single sideband modulator," Opt. Lett. 32, 3152-3154 (2007).

[21] M. Sagues, and A. Loayssa, "Orthogonally polarized optical single sideband modulation for microwave photonics processing using stimulated Brillouin scattering," Opt. Express 18, 2290622914 (2010).

[22] W. Li, N. H. Zhu, and L. X. Wang, "Perfectly Orthogonal Optical Single-Sideband Signal Generation Based on Stimulated Brillouin Scattering," IEEE Photonic Tech. L. 24, 751-753 (2012).

[23] W. L. Liu, M. Li, R. S. Guzzon, E. J. Norberg, J. S. Parker, M. Z. Lu, L. A. Coldren, and J. P. Yao, "A fully reconfigurable photonic integrated signal processor," Nat. Photonics 10, 190195 (2016)

[24] D. Marpaung, C. Roeloffzen, R. Heideman, A. Leinse, S. Sales, and J. Capmany, "Integrated microwave photonics," Laser Photonics Rev. 7, 506-538 (2013).

[25] X. Xu, J. Wu, M. X. Tan, T. G. Nguyen, S. T. Chu, B. E. Little, R. Morandotti, A. Mitchell, and D. J. Moss, "Orthogonally polarized RF optical single sideband generation and dual-channel equalization based on an integrated micro-ring resonator," IEEE J. Lightwave Technol. 36, 4808-4818 (2018).
[26] N. Cui, X. G. Zhang, Z. B. Zheng, H. Y. Xu, W. B. Zhang, X. F. Tang, L. X. Xi, Y. Y. Fang, and L. C. Li, "Two-parameterSOP and three-parameter-RSOP fiber channels: problem and solution for polarization demultiplexing using Stokes space," Opt. Express 26, 21170-21183 (2018).

[27] M. Peccianti, M. Ferrera, L. Razzari, R. Morandotti, B. E. Little, S. T. Chu, and D. J. Moss, "Subpicosecond optical pulse compression via an integrated nonlinear chirper," Opt. Express 18, 7625-7633 (2010).

[28] D. J. Moss, R. Morandotti, A. L. Gaeta, and M. Lipson, "New CMOS-compatible platforms based on silicon nitride and Hydex for nonlinear optics," Nat. Photonics 7, 597-607 (2013).

[29] M. Ferrera, L. Razzari, D. Duchesne, R. Morandotti, Z. Yang, M. Liscidini, J. E. Sipe, S. Chu, B. E. Little, and D. J. Moss, "Low-power continuous-wave nonlinear optics in doped silica glass integrated waveguide structures," Nat. Photonics 2, 737740 (2008).

[30] L. Razzari, D. Duchesne, M. Ferrera, R. Morandotti, S. Chu, B. E. Little, and D. J. Moss, "CMOS-compatible integrated optical hyper-parametric oscillator," Nat. Photonics 4, 41-45 (2010).

[31] A. Pasquazi et al., "Micro-Combs: A Novel Generation of Optical Sources", Physics Reports, 10, 1006 (2017).

[32] M. Kues, C. Reimer, B. Wetzel, P. Roztocki, B. E. Little, S. T. Chu, T. Hansson, E. A. Viktorov, D. J. Moss, and R. Morandotti, "Passively mode-locked laser with an ultra-narrow spectral width," Nat. Photonics. 11, 159-162 (2017).

[33] Z. Z. Lu, W. Q. Wang, W. F. Zhang, M. L. Liu, L. R. Wang, S. T. Chu, B. E. Little, J. G. Zhao, P. Xie, X. Y. Wang, and W. Zhao, "Raman self-frequency-shift of soliton crystal in a high index doped silica micro-ring resonator [Invited]," Opt. Mater. Express 8, 2662-2669 (2018).

[34] X. Xu, J. Y. Wu, T. G. Nguyen, T. Moein, S. T. Chu, B. E. Little, R. Morandotti, A. Mitchell, and D. J. Moss, "Broadband RF Channelizer based on an Integrated Optical Frequency Kerr Comb Source," IEEE J. Lightwave Technol. 36, 4519-4526 (2018).

[35] X. Xu, J. Wu, T. G. Nguyen, T. Moein, S. T. Chu, B. E. Little, R. Morandotti, A. Mitchell, and D. J. Moss, "Photonic microwave true time delays for phased array antennas using a 49GHz FSR integrated optical micro-comb source," Photonics Research 6, 30-36 (2018).

[36] A. Pasquazi, M. Peccianti, B. E. Little, S. T. Chu, D. J. Moss, and R. Morandotti, "Stable, dual mode, high repetition rate mode-locked laser based on a micro-ring resonator," Opt. Express 20, 27355-27362 (2012).

[37] X. Xu, J. Wu, M. Shoeiby, T. G. Nguyen, S. T. Chu, B. E. Little, R. Morandotti, A. Mitchell, and D. J. Moss, "Reconfigurable broadband microwave photonic intensity differentiator based on an integrated optical frequency comb source," APL Photonics 2, 096104 (2017).

[38] A. Pasquazi, L. Caspani, M. Peccianti, M. Clerici, M. Ferrera, L. Razzari, D. Duchesne, B. E. Little, S. T. Chu, D. J. Moss, and R. Morandotti, "Self-locked optical parametric oscillation in a CMOS compatible micro-ring resonator: a route to robust optical frequency comb generation on a chip," Opt. Express 21, 1333313341 (2013).

[39] X. Xu, J. Wu, T. G. Nguyen, M. Shoeiby, S. T. Chu, B. E. Little, R. Morandotti, A. Mitchell, and D. J. Moss, "Advanced 
$\mathrm{RF}$ and microwave functions based on an integrated optical frequency comb source," Opt. Express 26, 2569-2583 (2018).

[40] D.Duchesne, M.Ferrera, L.Razzari, R.Morandotti, S.Chu, B.Little, and D. J. Moss, "Efficient self-phase modulation in low loss, high index doped silica glass integrated waveguides", Optics Express 17, 1865 (2009).

[41] Q. F. Xu, and M. Lipson, "All-optical logic based on silicon micro-ring resonators," Opt. Express 15, 924-929 (2007).

[42] A. Godbole, P. P. Dali, V. Janyani, T. Tanabe, and G. Singh, "All Optical Scalable Logic Gates Using Si3N4 Microring Resonators," IEEE J. Sel. Top. Quant. 22, 5900308 (2016).

[43] X. X. Xue, Y. Xuan, C. Wang, P. H. Wang, Y. Liu, B. Niu, D. E. Leaird, M. H. Qi, and A. M. Weiner, "Thermal tuning of Kerr frequency combs in silicon nitride microring resonators," Opt. Express 24, 687-698 (2016).

[44] B. Gerislioglu, A. Ahmadivand, M. Karabiyik, R. Sinha, and N. Pala, "VO2-Based Reconfigurable Antenna Platform with Addressable Microheater Matrix," Adv. Electron. Mater. 3, 1700170(2017).

[45] C. Akcay, P. Parrein, and J. P. Rolland, "Estimation of longitudinal resolution in optical coherence imaging," Appl. Optics 41, 5256-5262 (2002).

[46] A. Pasquazi, R. Ahmad, M. Rochette, M. Lamont, B. E. Little, S. T. Chu, R. Morandotti, and D. J. Moss, "All-optical wavelength conversion in an integrated ring resonator," Opt. Express 18, 3858-3863 (2010).

[47] J. Wu, T. Moein, X. Y. Xu, and D. J. Moss, "Advanced photonic filters based on cascaded Sagnac loop reflector resonators in silicon-on-insulator nanowires," APL Photonics 3, 046102 (2018).

[48] B. E. Little, S. T. Chu, H. A. Haus, J. Foresi, and J. P. Laine, "Micro-ring resonator channel dropping filters," J. Lightwave Technol. 15, 998-1005 (1997).

[49] J. Wu, T. Moein, X. Y. Xu, G. H. Ren, A. Mitchell, and D. J. Moss, "Micro-ring resonator quality factor enhancement via an integrated Fabry-Perot cavity," APL Photonics 2, 056103 (2017).

[50] J. Wu, P. Cao, T. Pan, Y. Yang, C. Qiu, C. Tremblay, and Y. $\mathrm{K}$. Su, "Compact on-chip $1 \times 2$ wavelength selective switch based on silicon microring resonator with nested pairs of subrings," Photonics Res. 3, 9-14 (2015).

[51] H. Arianfard, J. Wu, S. Juodkazis, and D. J. Moss, “Advanced Multi-Functional Integrated Photonic Filters Based on Coupled Sagnac Loop Reflectors", Journal of Lightwave Technology, Vol. 39, Issue: 5, pp. 1400-1408 (2021). DOI: 10.1109/JLT.2020.3037559.

[52] H. Arianfard, J. Wu, S. Juodkazis and D. J. Moss, "Three Waveguide Coupled Sagnac Loop Reflectors for Advanced Spectral Engineering", Journal of Lightwave Technology, Vol. 39, Early Access (2021). DOI: 10.1109/JLT.2021.3066256.

[53] A. Dutt, C. Joshi, X. C. Ji, J. Cardenas, Y. Okawachi, K. Luke, A. L. Gaeta, and M. Lipson, "On-chip dual-comb source for spectroscopy," Sci. Adv. 4, e1701858 (2018).

[54] M.R.E. Lamont, M. Rochette, D.J. Moss, B.J. Eggleton, "Two-photon absorption effects on self-phase-modulationbased 2R optical regeneration", IEEE Photon. Technol. Lett. 18 (10), 1185 (2006).

[55] A. Tuniz, G. Brawley, D.J. Moss, B.J. Eggleton, “Two-photon absorption effects on Raman gain in single mode As2Se3 chalcogenide glass fiber”, Opt. Express 16 (22), 18524 (2008).
[56] Mengxi Tan, X. Xu, J. Wu, T. G. Nguyen, S. T. Chu, B. E. Little, R. Morandotti, A. Mitchell, and David J. Moss, "Photonic Radio Frequency Channelizers based on Kerr Optical Micro-combs", Journal of Semiconductors vol. 42 (4), 041302 (2021).

[57] H.Bao, L.Olivieri, M.Rowley, S.T. Chu, B.E. Little, R.Morandotti, D.J. Moss, J.S.T. Gongora, M.Peccianti and A.Pasquazi, "Laser Cavity Solitons and Turing Patterns in Microresonator Filtered Lasers: properties and perspectives", Paper No. LA203-5, Paper No. 11672-5, SPIE LASE, SPIE Photonics West, San Francisco CA March 6-11 (2021). DOI: $10.1117 / 12.2576645$

[58] Mengxi Tan, X. Xu, J. Wu, A. Boes, T. G. Nguyen, S. T. Chu, B. E. Little, R. Morandotti, A. Mitchell, and David J. Moss, "Advanced microwave signal generation and processing with soliton crystal microcombs", or "Photonic convolutional accelerator and neural network in the Tera-OPs regime based on Kerr microcombs", Paper No. 11689-38, PW21O-OE201-67, Integrated Optics: Devices, Materials, and Technologies XXV, SPIE Photonics West, San Francisco CA March 6-11 (2021). DOI: $10.1117 / 12.2584017$

[59] Mengxi Tan, Bill Corcoran, Xingyuan Xu, Andrew Boes, Jiayang Wu, Thach Nguyen, Sai T. Chu, Brent E. Little, Roberto Morandotti, Arnan Mitchell, and David J. Moss, "Optical data transmission at 40 Terabits/s with a Kerr soliton crystal microcomb", Paper No.11713-8, PW21O-OE803-23, Next-Generation Optical Communication: Components, SubSystems, and Systems X, SPIE Photonics West, San Francisco CA March 6-11 (2021). DOI:10.1117/12.2584014

[60] Mengxi Tan, X. Xu, J. Wu, A. Boes, T. G. Nguyen, S. T. Chu, B. E. Little, R. Morandotti, A. Mitchell, and David J. Moss, "RF and microwave photonic, fractional differentiation, integration, and Hilbert transforms based on Kerr microcombs", Paper No. 11713-16, PW21O-OE803-24, NextGeneration Optical Communication: Components, SubSystems, and Systems X, SPIE Photonics West, San Francisco CA March 6-11 (2021). DOI:10.1117/12.2584018

[61] Mengxi Tan, X. Xu, J. Wu, A. Boes, T. G. Nguyen, S. T. Chu, B. E. Little, R. Morandotti, A. Mitchell, and David J. Moss, "Broadband photonic RF channelizer with 90 channels based on a soliton crystal microcomb", or "Photonic microwave and RF channelizers based on Kerr micro-combs", Paper No. 11685-22, PW21O-OE106-49, Terahertz, RF, Millimeter, and Submillimeter-Wave Technology and Applications XIV, SPIE Photonics West, San Francisco CA March 6-11 (2021). DOI: $10.1117 / 12.2584015$

[62] X. Xu, M. Tan, J. Wu, S. T. Chu, B. E. Little, R. Morandotti, A. Mitchell, B. Corcoran, D. Hicks, and D. J. Moss, "Photonic perceptron based on a Kerr microcomb for scalable high speed optical neural networks", IEEE Topical Meeting on Microwave Photonics (MPW), pp. 220-224, Matsue, Japan, November 2426, 2020. DOI: 10.23919/MWP48676.2020.9314409

[63] Mengxi Tan, Bill Corcoran, Xingyuan Xu, Andrew Boes, Jiayang Wu, Thach Nguyen, S.T. Chu, B. E. Little, Roberto Morandotti, Arnan Mitchell, and David J. Moss, "Ultra-high bandwidth optical data transmission with a microcomb", IEEE Topical Meeting on Microwave Photonics (MPW), pp. 78-82. Virtual Conf., Matsue, Japan, November 24-26, 2020. DOI: 10.23919/MWP48676.2020.9314476 
[64] M. Tan, X. Xu, J. Wu, R. Morandotti, A. Mitchell, and D. J. Moss, "RF and microwave high bandwidth signal processing based on Kerr Micro-combs", Advances in Physics X, VOL. 6, NO. 1, 1838946 (2020). DOI:10.1080/23746149.2020.1838946.

[65] Mengxi Tan, Xingyuan Xu, Jiayang Wu, Thach G. Nguyen, Sai T. Chu, Brent E. Little, Roberto Morandotti, Arnan Mitchell, and David J. Moss, "Photonic Radio Frequency Channelizers based on Kerr Micro-combs and Integrated Microring Resonators", JOSarXiv.202010.0002.

[66] Mengxi Tan, Xingyuan Xu, David Moss "Tunable Broadband RF Photonic Fractional Hilbert Transformer Based on a Soliton Crystal Microcomb", Preprints, DOI:10.20944/preprints202104.0162.v1

[67] Mengxi Tan, X. Xu, J. Wu, T. G. Nguyen, S. T. Chu, B. E. Little, R. Morandotti, A. Mitchell, and David J. Moss, "Orthogonally polarized Photonic Radio Frequency single sideband generation with integrated micro-ring resonators", Journal of Semiconductors 42 (4), 041305 (2021). DOI: 10.1088/1674-4926/42/4/041305.

[68] Mengxi Tan, Xingyuan Xu, Jiayang Wu, David J. Moss, "High bandwidth temporal RF photonic signal processing with Kerr micro-combs: integration, fractional differentiation and Hilbert transforms", arXiv:2103.03674 [physics.app-ph] (2021).

[69] D.J. Moss, "Optimization of Optical Filters based on Integrated Coupled Sagnac Loop Reflectors", Research Square (2021). DOI: https://doi.org/10.21203/rs.3.rs-478204/v1

[70] Mengxi Tan, Xingyuan Xu, David J. Moss, "Integral order photonic RF and microwave signal processors based on Kerr micro-combs", Research Square

https://www.researchsquare.com/article/rs-441212/v1 (2021). DOI:10.21203/rs.3.rs-441212/v1.

[71] D.J. Moss, "Integral Order RF and Microwave Photonic Based Signal Processors Using Kerr Soliton Crystal Microcombs.” OSF Preprints, 31 May 2021. DOI:10.31219/osf.io/fvzw6 (2021).

[72] Mengxi Tan, Xingyuan Xu, Jiayang Wu, Bill Corcoran, Andreas Boes, Thach G. Nguyen, Sai T. Chu, Brent E. Little, Roberto Morandotti, Arthur Lowery, Arnan Mitchell, and David J. Moss, "Highly reconfigurable RF photonic fractional Hilbert transformer", Journal of Lightwave Technology, Vol. 39, Early Access (2021). DOI:10.1109/JLT.2021.3101816.

[73] Mengxi Tan, Xingyuan Xu, Jiayang Wu, Roberto Morandotti, Arnan Mitchell, and David J. Moss, "RF and microwave photonic temporal signal processing with Kerr micro-combs", Advances in Physics X, VOL. 6, NO. 1, 1838946 (2021). DOI:10.1080/23746149.2020.1838946.

[74] T. G. Nguyen et al., "Integrated frequency comb source-based Hilbert transformer for wideband microwave photonic phase analysis," Opt. Express, vol. 23, no. 17, pp. 22087-22097, Aug. 2015.

[75] X. Xue, et al., "Programmable single-bandpass photonic RF filter based on a Kerr comb from a microring," Journal of Lightwave Technol., vol. 32, no. 20, pp. 3557-3565, Oct. 2014.

[76] X. Xu, J. Wu, M. Shoeiby, T. G. Nguyen, S. T. Chu, B. E. Little, R. Morandotti, A. Mitchell, and D. J. Moss,

"Reconfigurable broadband microwave photonic intensity differentiator based on an integrated optical frequency comb source," APL Photonics, vol. 2, no. 9, 096104, Sep. 2017.
[77] X. Xu, M. Tan, J. Wu, R. Morandotti, A. Mitchell, and D. J. Moss, "Microcomb-based photonic RF signal processing", IEEE Photonics Technology Letters, vol. 31 no. 23 1854-1857, 2019.

[78] X. Xu, et al., "Advanced RF and microwave functions based on an integrated optical frequency comb source," Opt. Express, vol. 26, no. 3, pp. 2569-2583, Feb. 2018.

[79] X. Xue, et al., "Microcomb-based true-time-delay network for microwave beamforming with arbitrary beam pattern control," Journal of Lightwave Technology, vol. 36, no. 12, pp. 23122321, Jun. 2018

[80] X. Xu, et al., "Broadband RF channelizer based on an integrated optical frequency Kerr comb source," Journal of Lightwave Technology, vol. 36, no. 19, pp. 4519-4526, 2018.

[81] X. Xu, et al., "Continuously tunable orthogonally polarized RF optical single sideband generator based on micro-ring resonators," Journal of Optics, vol. 20, no. 11, 115701. 2018.

[82] X. Xu, et al., "Orthogonally polarized RF optical single sideband generation and dual-channel equalization based on an integrated microring resonator," Journal of Lightwave Technology, vol. 36, no. 20, pp. 4808-4818. 2018.

[83] X. Xu, et al., "Photonic microwave true time delays for phased array antennas using a $49 \mathrm{GHz}$ FSR integrated optical micro-comb source," Photonics Res, vol. 6, no. 5, pp. B30-B36, 2018.

[84] X. Xu, et al., "Advanced adaptive photonic RF filters with 80 taps based on an integrated optical micro-comb source," Journal of Lightwave Technology, vol. 37, no. 4, pp. 1288-1295, 2019.

[85] W. Liang, et al., "High spectral purity Kerr frequency comb radio frequency photonic oscillator," Nature Communications, vol. 6 pp. 7957. 2015.

[86] X. Xu et al., Broadband microwave frequency conversion based on an integrated optical micro-comb source", Journal of Lightwave Technology, vol. 38 no. 2, pp. 332-338. 2020.

[87] M. Tan et al., "Photonic RF and microwave filters based on $49 \mathrm{GHz}$ and $200 \mathrm{GHz}$ Kerr microcombs", Optics Comm. vol. 465, Article: 125563, Feb. 22. 2020.

[88] X. Xu et al., "Broadband photonic RF channelizer with 90 channels based on a soliton crystal microcomb", Journal of Lightwave Technology, Vol. 38, no. 18, pp.5116 - 5121, 2020. doi: 10.1109/JLT.2020.2997699.

[89] X. Xu et al., "Photonic RF and microwave integrator with soliton crystal microcombs", IEEE Transactions on Circuits and Systems II: Express Briefs, Vol. 67 (12), 3582-3586. 2020. DOI:10.1109/TCSII.2020.2995682.

[90] X. Xu et al., "Photonic RF phase-encoded signal generation with a microcomb source", Journal of Lightwave Technology, vol. 38 , no. 7, pp. 1722-1727 (2020).

[91] X. Xu et al., "High performance RF filters via bandwidth scaling with Kerr micro-combs," APL Photonics, vol. 4, no. 2, pp. 026102. 2019.

[92] M. Tan et al., "Microwave and RF photonic fractional Hilbert transformer based on a $50 \mathrm{GHz}$ Kerr micro-comb", Journal of Lightwave Technology, vol. 37, no. 24, pp. 6097 - 6104, 2019.

[93] M. Tan et al., "RF and microwave fractional differentiator based on photonics", IEEE Transactions on Circuits and Systems: Express Briefs, Vol. 67, No. 11, pp. 2767 - 2771 (2020). DOI:10.1109/TCSII.2020.2965158. 
[94] M. Tan et al., "Photonic RF arbitrary waveform generator based on a soliton crystal micro-comb source", Journal of Lightwave Technology, Vol. 38, No. 22, pp. 6221-6226, Oct 22 (2020). DOI: 10.1109/JLT.2020.3009655.

[95] M. Tan, X. Xu, J. Wu, R. Morandotti, A. Mitchell, and D. J. Moss, "RF and microwave high bandwidth signal processing based on Kerr Micro-combs", Advances in Physics X, VOL. 6, NO. 1, 1838946 (2021). DOI:10.1080/23746149.2020.1838946.

[96] L. Razzari, D. Duchesne, M. Ferrera, et al., "CMOScompatible integrated optical hyper-parametric oscillator," Nature Photonics, vol. 4, no. 1, pp. 41-45, 2010.

[97] D. J. Moss, R. Morandotti, A. L. Gaeta, et al., "New CMOScompatible platforms based on silicon nitride and Hydex for nonlinear optics," Nature Photonics, vol. 7, no. 8, pp. 597-607, 2013.

[98] M. Ferrera, L. Razzari, D. Duchesne, et al., "Low-power continuous-wave nonlinear optics in doped silica glass integrated waveguide structures," Nature Photonics, vol. 2, no. 12, pp. 737-740, 2008.

[99] A. Pasquazi, et al., "Sub-picosecond phase-sensitive optical pulse characterization on a chip", Nature Photonics, vol. 5, no. 10, pp. 618-623 (2011). DOI: 10.1038/nphoton.2011.199.

[100] D. Duchesne, M. Peccianti, M. R. E. Lamont, et al., "Supercontinuum generation in a high index doped silica glass spiral waveguide," Optics Express, vol. 18, no, 2, pp. 923-930, 2010.

[101] M. Ferrera, et al., "On-chip CMOS-compatible all-optical integrator", Nature Communications, vol. 1, Article 29, 2010. DOI: $10.1038 /$ ncomms 1028

[102] A. Pasquazi, et al., "All-optical wavelength conversion in an integrated ring resonator," Optics Express, vol. 18, no. 4, pp. 3858-3863, 2010.

[103] A. Pasquazi, Y. Park, J. Azana, et al., "Efficient wavelength conversion and net parametric gain via Four Wave Mixing in a high index doped silica waveguide," Optics Express, vol. 18, no. 8, pp. 7634-7641, 2010.

[104] M. Peccianti, M. Ferrera, L. Razzari, et al., "Subpicosecond optical pulse compression via an integrated nonlinear chirper," Optics Express, vol. 18, no. 8, pp. 7625-7633, 2010.

[105] D. Duchesne, M. Ferrera, L. Razzari, et al., "Efficient selfphase modulation in low loss, high index doped silica glass integrated waveguides," Optics Express, vol. 17, no. 3, pp. 1865-1870, 2009.

[106] B. Corcoran, et al., "Ultra-dense optical data transmission over standard fiber with a single chip source", Nature Communications, vol. 11, Article:2568, 2020. DOI:10.1038/s41467-020-16265-x.

[107] X. Xu, et al., "Photonic perceptron based on a Kerr microcomb for scalable high speed optical neural networks", Laser and Photonics Reviews, vol. 14, no. 8, 2000070, 2020. DOI:10.1002/lpor.202000070.

[108] X. Xu, et al., "11 TOPs photonic convolutional accelerator for optical neural networks", Nature, Vol. 589, no. 7840, pp. 44-51. 2021.

[109] A. Pasquazi, M. Peccianti, L. Razzari, D. J. Moss, S. Coen, M. Erkintalo, Y. K. Chembo, T. Hansson, S. Wabnitz, P. Del'Haye, X. X. Xue, A. M. Weiner, and R. Morandotti, "Micro-combs: A novel generation of optical sources," Physics Reports, vol. 729, pp. 1-81, Jan 27. 2018.
[110] M. Peccianti, et al., "Demonstration of an ultrafast nonlinear microcavity modelocked laser", Nature Communications, vol. 3, pp. 765, 2012. DOI:10.1038/ncomms 1762

[111] M. Kues, et al., "Passively modelocked laser with an ultranarrow spectral width", Nature Photonics, vol. 11, no. 3, pp. 159, 2017. DOI:10.1038/nphoton.2016.271

[112] A. Pasquazi, L. Caspani, M. Peccianti, et al., "Self-locked optical parametric oscillation in a CMOS compatible microring resonator: a route to robust optical frequency comb generation on a chip," Optics Express, vol. 21, no. 11, pp. 13333-13341, 2013.

[113] A. Pasquazi, M. Peccianti, B. E. Little, et al., "Stable, dual mode, high repetition rate mode-locked laser based on a microring resonator," Optics Express, vol. 20, no. 24, pp. 27355-27362, 2012.

[114] C. Reimer, L. Caspani, M. Clerici, et al., "Integrated frequency comb source of heralded single photons," Optics Express, vol. 22, no. 6, pp. 6535-6546, 2014.

[115] C. Reimer, et al., "Cross-polarized photon-pair generation and bi-chromatically pumped optical parametric oscillation on a chip", Nature Communications, vol. 6, Article 8236, 2015. DOI: $10.1038 /$ ncomms 9236

[116] L. Caspani, C. Reimer, M. Kues, et al., "Multifrequency sources of quantum correlated photon pairs on-chip: a path toward integrated Quantum Frequency Combs," Nanophotonics, vol. 5, no. 2, pp. 351-362, 2016.

[117] C. Reimer, M. Kues, P. Roztocki, B. Wetzel, F. Grazioso, B. E. Little, S. T. Chu, T. Johnston, Y. Bromberg, L. Caspani, D. J. Moss, and R. Morandotti, "Generation of multiphoton entangled quantum states by means of integrated frequency combs," Science, vol. 351, no. 6278, pp. 1176-1180, 2016.

[118] M. Kues, et al., "On-chip generation of high-dimensional entangled quantum states and their coherent control", Nature, vol. 546, no. 7660, pp. 622-626, 2017.

[119] P. Roztocki, M. Kues, C. Reimer, B. Wetzel, S. Sciara, Y. Zhang, A. Cino, B. E. Little, S. T. Chu, D. J. Moss, and R. Morandotti, "Practical system for the generation of pulsed quantum frequency combs," Optics Express, vol. 25, no. 16, pp. 18940-18949, 2017.

[120] Y. Zhang, et al., "Induced photon correlations through superposition of two four-wave mixing processes in integrated cavities", Laser and Photonics Reviews, vol. 14, no. 7, pp. 2000128, 2020. DOI: 10.1002/lpor.202000128

[121] M. Kues, C. Reimer, A. Weiner, J. Lukens, W. Munro, D. J. Moss, and R. Morandotti, "Quantum Optical Micro-combs", Nature Photonics, vol. 13, no.3, pp. 170-179, 2019.

[122] C. Reimer, et al.,"High-dimensional one-way quantum processing implemented on d-level cluster states", Nature Physics, vol. 15, no.2, pp. 148-153, 2019. 